\title{
APPROXIMATE SOLUTIONS OF BOUNDARY VALUE PROBLEMS IN POTENTIAL THEORY WITH APPLICA- TIONS TO ELECTROSTATICS AND ELASTOSTATICS
}

\author{
by W. E. WILLIAMS \\ (Received 15th December 1963)
}

\section{Summary}

A simple approximate formula is obtained for the capacity of the condenser formed by a " small" conductor placed inside a much larger one. The formula involves a constant whose choice is, to a certain extent, arbitrary and it is shown that, for problems involving spheroids inside cylinders and between parallel plates, the constant may be found in a simple fashion so as to give very accurate results. A similar formula is obtained for the loss in potential energy due to a crack or cavity in a circular beam or a thick plate. For the particular cases of the boundary value problems considered which have been treated by other means very close numerical agreement is obtained between those results and ones deduced in the present paper.

\section{Introduction}

Recently, several papers have appeared treating particular cases of the general problem of determining the electrostatic potential in the region between two conductors $S_{1}$ and $S_{2}$ with the potential given on $S_{1}$ and $S_{2} .(1,2,3,4$.) The methods used in the above references are moderately elaborate and consist in reducing the problem to the solution of an infinite set of equations $(1,2)$ or to a Fredholm integral equation of the second kind $(3,4)$. Furthermore, in order to make any appreciable progress with these methods it has been found necessary to assume that the minimum distance $(b)$ between a point of $S_{1}$ and a point of $S_{2}$ is much greater than the maximum distance $(a)$ between two points of $S_{1}$.

In view of the complexity of the boundary value problem, even for simple surfaces $S_{1}$ and $S_{2}$, it seems inevitable that elaborate methods of the above type will have to be used in order to obtain a complete solution. It does seem, however, to be of interest to examine whether it is possible to obtain some results of physical significance without much elaborate calculation. The present work is concerned with this particular aspect of the general electrostatic problem and of similar problems in elastostatics. For the electrostatic problem it transpires that a simple and, in some cases, very accurate form can be obtained for the capacity $C$ of the conductor formed by $S_{1}$ and $S_{2}$.

E.M.S.- $-G$ 
In Section 2 the electrostatic boundary value problem is formulated as a Fredholm integral equation of the first kind and it is shown that, neglecting $O\left(a^{2} / b^{2}\right)$, a solution can be obtained in terms of the solution for the corresponding problem in the absence of $S_{2}$. To this order of accuracy a simple relationship is then derived between $C$ and $C_{0}$, the capacity of $S_{1}$ in free space. The relationship obtained is valid for arbitrary $S_{1}$ and $S_{2}$ and, neglecting $O\left(a^{2} / b^{2}\right)$, is precisely that which would have been obtained by a direct perturbation approach. The relationship involves a constant $A$ and there is a certain degree of freedom available as to the most appropriate value of $A$ to choose. It is shown that, when $S_{2}$ is an infinite cylinder or a pair of parallel planes, it is possible to choose $A$ so that $C / C_{0}$ is determined correct including terms of $O\left(a^{2} / b^{2}\right)$. Furthermore, for the particular cases of spheroids in circular cylinders or between parallel plates it is shown that $A$ can be chosen in a simple way so that the error in the formula obtained for $C / C_{0}$ is $O\left(a^{6} / b^{6}\right)$.

In Sections 4, 5, various problems for cracks and cavities in circular beams and thick plates are considered. For the case of an axi-symmetric crack in a circular cylinder subjected to an axial couple simple expressions involving one constant are found for the effect of the crack on the strain energy and the angle of twist. The error in these expressions is $O\left(a^{7} / b^{7}\right)$ for arbitrary cracks but for spheroidal cavities it is shown that the constant may be chosen so that the error is $O\left(a^{14} / b^{14}\right)$. For the particular case of a penny shaped crack a power series in $a / b$ is derived for the strain energy and this agrees with a corresponding result obtained by Collins (5). For a spherical cavity in a cylindrical beam the numerical values obtained for the angle of twist are in very close agreement with those of Ling (6). Problems involving spheroidal cavities in a thick plate under torsion are also examined. The problem of an oblate spheroidal cavity rotated as a rigid body in a thick plate with fixed plane surfaces is considered briefly and an expression given for the applied couple necessary to give the prescribed rotation. The numerical value of the applied couple agrees exactly with that obtained by Fox (7) for the case when the sphere radius is half that of the cylinder.

\section{Basic Formula}

The problem considered is that of determining the electrostatic potential in the region between two conductors $S_{1}$ and $S_{2}, S_{1}$ being completely contained in $S_{2}$ and kept at unit potential, the potential on $S_{2}$ being zero. The maximum distance between two points on $S_{1}$ will be denoted by $a$ and the minimum distance between a point of $S_{1}$ and a point of $S_{2}$ will be denoted by $b$. It will also be assumed that $(a / b)$ is small compared to unity.

The potential $V$ will be of the form

$$
V=\int_{S_{1}} G(P, Q) \sigma(P) d S,
$$

where $Q$ is an arbitrary point in the region between the conductors, $P$ is a point 
of $S_{1}, \sigma(P)$ denotes the charge density at $P$ and $G(P, Q)$ is the Green's function vanishing on $S_{2}$. From equation (1) it follows that the boundary value problem reduces to the solution of

$$
1=\int_{S_{1}} G(P, Q) \sigma(P) d S, \quad Q \text { on } S_{1} .
$$

$G(P, Q)$ can be written as the sum of the free space Green's function $G_{0}(P, Q)$ and a perturbation term $G_{1}(P, Q)$ and hence equation (2) becomes

$$
1=\int_{S_{1}} G_{0}(P, Q) \sigma(P) d S+\int_{S_{1}} G_{1}(P, Q) \sigma(P) d S, \quad Q \text { on } S_{1} .
$$

Physically the second integral on the right hand side of equation (3) represents the effect of the conductor $S_{2}$ on the potential on $S_{1}$ and hence it will be of $O(\varepsilon)$ times the first integral, where $\varepsilon=a / b$. It will now be assumed that, on $S_{1}, G_{1}$ can be written as $A+G_{2}(P, Q)$ where $A$ is a constant and $G$ is $O(A \varepsilon)$. Clearly this can always be done as one possible value for $A$ is the value of $G_{1}(P, Q)$ for any arbitrary pair of points $P$ and $Q$ on $S_{1} . \quad G_{2}$ would then be the deviation of $G_{1}$ on $S$ from this constant value and would thus be of the stated order. Hence

$1=\int_{S_{1}} G_{0}(P, Q) \sigma(P) d S+A \int_{S_{1}} \sigma(P) d S+\int_{S_{1}} G_{2}(P, Q) \sigma(P) d S, Q$ on $S_{1}$.

Equation (4) can be re-written as

where

$$
1=\int_{S_{1}} G_{0}(P, Q) \sigma^{\prime}(P) d S+\int_{S_{1}} G_{2}(P, Q) \sigma^{\prime}(P) d S, \quad Q \text { on } S_{1},
$$

It follows from equation (6) that

$$
\sigma^{\prime}=\frac{\sigma}{1-A \int_{S_{1}} \sigma d S}
$$

$$
\int_{S_{1}} \sigma d S=\frac{\int_{S_{1}} \sigma^{\prime} d S}{1+A \int_{S_{1}} \sigma^{\prime} d S} .
$$

The above arguments show that the second integral on the right hand side of equation (5) is $O\left(\varepsilon^{2}\right)$ times the first. Hence, neglecting terms of this order, $\sigma^{\prime}$ is the electrostatic charge density on $S_{1}$ when raised to unit potential in free space. It will be assumed that $S_{1}$ is such that the solution to this problem can be found. It now follows from equation (7) that the capacity $C$ of the conductor formed by $S_{1}$ and $S_{2}$ is given by

$$
\frac{C}{C_{0}}=\left(1+A C_{0}\right)^{-1}+O\left(\varepsilon^{2}\right)
$$


Equation (8) is the basic equation of the present paper and all the electrostatic results are derived directly from it. Equation (8) shows that

$$
C / C_{0}=1-A C_{0}+O\left(\varepsilon^{2}\right)
$$

and if $A$ is interpreted as the value of $G(P, Q)$ for any pair of points $P, Q$ on $S_{1}$ this result for the capacity is precisely that which would have been obtained by a simple perturbation procedure. The usefulness of equation (8) in determining the electrostatic capacity lies in the fact that in many situations it is possible to show that, by a suitable choice of $A$, the error term can be shown to be $O\left(\varepsilon^{3}\right)$. This type of situation occurs when $G_{2}$ is $O\left(\varepsilon^{2} A\right)$ and is best illustrated by considering two particular cases which give rise to this type of situation.

The first case is that when $S_{2}$ is a circular cylinder of radius $c . G_{1}$ then has the form (4)

$$
G_{1}=\sum_{r=0}^{\infty}\left(2-\delta_{0 r}\right) G_{1}^{(r)}\left(\rho_{1}, \rho_{2}, z_{1}, z_{2}\right) \cos r\left(\phi_{1}-\phi_{2}\right)
$$

where

$$
G_{1}^{(r)}=-\frac{2}{\pi} \int_{0}^{\infty} \frac{I_{r}\left(p \rho_{1}\right) I_{r}\left(p \rho_{2}\right) K_{r}(p c)}{I_{r}(p c)} \cos p\left(z_{1}-z_{2}\right) d p,
$$

and $\delta_{0 r}$ is the Kronecker delta. $\left(\rho_{1}, z_{1}, \phi_{1}\right)$ are the cylindrical polar coordinates of $P$ referred to a coordinate system whose axis coincides with that of the cylinder, $\left(\rho_{2}, z, \phi_{2}\right)$ are the corresponding coordinates for $Q$. The corresponding result for the case when $S_{2}$ is a pair of parallel plates a distance $2 d$ apart is also given by equation (9) where $G_{1}$ is now defined by

$G_{1}^{(r)}=\int_{0}^{\infty} \frac{e^{-p d}}{\sinh 2 p d}\left[e^{-p d} \cosh p\left(z_{1}-z_{2}\right)-e^{p d} \cosh p\left(z_{1}+z_{2}\right)\right] J_{r}\left(p \rho_{1}\right) J_{r}\left(p \rho_{2}\right) d p$.

The symbols $\rho_{1}, z_{1}, \phi_{1}$, etc., have the same meaning as in equation (10) except that the $z$-axis is now perpendicular to the plane of the plates with the origin midway between the plates.

It follows from equation (10) that, when $S_{2}$ is a circular cylinder, the deviation of $G_{1}$ on $S_{1}$ from the value of $G_{1}(P, P)$, where $P$ is on the axis of the cylinder, is of the second order. Hence if $A$ is defined by

$$
A=-\frac{2}{\pi c} I(0)
$$

where

$$
\frac{I(2 m)}{2 m+1}=\int_{0}^{\infty} \frac{u^{2 m} K_{0}(u)}{I_{0}(u)} d u
$$

it follows that the error term in equation (8) is $O\left(\varepsilon^{3}\right)$. Hence for a conductor $S_{1}$ placed near the axis of a circular cylinder the capacity $C$ of the combined 
conductor is given by

$$
\frac{C}{C_{0}}=\left(1-\frac{2}{\pi c} C_{0} I(0)\right)^{-1}+O\left(\varepsilon^{3}\right) .
$$

The known solutions (4) for a disc or a cap in a circular cylinder are of the above form. [ $\pi^{2}$ in equation (74) of (4) should be replaced by $\pi$.]

It follows similarly from equation (11) that, if $A$ is defined as $G_{1}(P, P)$ where $P$ is any point on the plane $z=0$, the error term in equation (8) is again $O\left(\varepsilon^{3}\right)$. Hence for a conductor $S_{1}$ in the neighbourhood of the plane $z=0$

$$
\frac{C}{C_{0}}=\left(1-\frac{C_{0}}{d} \log 2\right)^{-1}+O\left(\varepsilon^{3}\right)
$$

The known solutions for a cap and a disc between two parallel planes satisfy equation (14).

It has thus been shown that for certain conductors $S_{2}$ the result of equation (8) is somewhat more accurate than one would have expected initially. For certain bodies $S_{1}$ such as spheres or spheroids it is possible to show that $A$ can be chosen in a simple fashion so that the error in equation (8) is $O\left(\varepsilon^{6}\right)$. These particular cases will now be discussed.

\section{Problems involving spheroids}

The general approach will be illustrated by considering the particular case of a sphere of radius $a$ placed with its centre $O$ on the axis of a circular cylinder of radius $c$. Since the problem considered is axi-symmetric it follows that the only contribution to the second integral in equation (3) will come from $G_{1}^{(0)}$. It also follows from the form of $G_{1}^{(0)}$ given in equation (10) and the general theory of solutions of Laplace's equation that, on the sphere

$$
G_{1}^{(0)}(P, Q)=\frac{-1}{c} \operatorname{Re}\left\{\sum_{n=0}^{\infty} \varepsilon_{1}^{n} A_{n} P_{n}\left(\cos \theta_{1}\right)\right\}\left\{\sum_{n=0}^{\infty} \varepsilon_{1}^{n} A_{n} P_{n}\left(\cos \theta_{2}\right)\right\} .
$$

In equation (15) $\theta_{1}$ and $\theta_{2}$ denotes the angles between $O z$ and $O P, O Q$, respectively, $\varepsilon_{1}$ denotes $a / c$ and the constants $A_{n}$ are dimensionless constants of order unity with $A_{2 n+1}$ being purely imaginary. From the arguments of Section 2 it follows that $\sigma^{\prime}=\sigma_{0}$ (a constant $)+O\left(\sigma_{0} \varepsilon_{1}^{3}\right)$, and it follows from this result, equation (14) and the orthogonality properties of the Legendre function that the second term on the right hand side of equation (5) has the form

$$
\frac{A_{0}}{c}\left\{\varepsilon_{1}^{2} A_{2} P_{2}\left(\cos \theta_{2}\right)+\varepsilon_{1}^{4} A_{4} P_{4}\left(\cos \theta_{2}\right)\right\} \int_{S_{1}} \sigma_{0} d S+O\left(\varepsilon_{1}^{6}\right),
$$

provided that $A=-A_{0}^{2} / c$. In this case the value of $A$ is again that given by equation (12). It thus follows that the terms of $O\left(\sigma_{0} \varepsilon_{1}^{2}\right)$ and $O\left(\sigma_{0} \varepsilon_{1}^{4}\right)$ in $\sigma^{\prime}$ will be proportional to $P_{2}\left(\cos \theta_{1}\right)$ and $P_{4}\left(\cos \theta_{1}\right)$ and hence make no contribution to $\int_{S_{1}} \sigma^{\prime} d S$. Hence equation (13) is valid with the $O\left(\varepsilon^{3}\right)$ term replaced by $O\left(\varepsilon^{6}\right)$ 
and equation (14) can also be shown to be valid to the same order of error. To this order of approximation equations (13) and (14) had previously been obtained by the author (4) and Collins (3) respectively, by different more elaborate methods.

The above analysis can now be extended almost immediately to cover the cases of oblate and prolate spheroids placed with their centres on the axis of a circular cylinder and with their axes of symmetry coinciding with that of the cylinder. The case of an oblate spheroid will be considered first. It is convenient to use an oblate spheroidal coordinate system such that the coordinates of $P$ and $Q$ are, respectively, $\left(\zeta_{1}, \xi_{1}, \phi_{1}\right),\left(\zeta_{2}, \xi_{2}, \phi_{2}\right)$ where the oblate coordinates are related to the corresponding cylindrical polar ones by the formulæ

$$
z_{i}=a e \xi_{i} \zeta_{i}, \quad \rho_{i}=a e\left\{\left(1-\xi_{i}^{2}\right)\left(1+\zeta_{i}^{2}\right)\right\}^{\frac{1}{2}}, \quad i=1,2 .
$$

$a$ denotes the semi major axis of the spheroid and $e$ the eccentricity of its elliptic cross-section.

It follows by an extension of the analysis for the sphere that equation (8) will again be valid with an error of $O\left(\varepsilon_{1}^{6}\right)$ if $A$ is taken to be the constant term in the expansion of $G_{1}^{0}$ on the spheroid as a series in $P_{n}\left(\xi_{1}\right), P_{n}\left(\xi_{2}\right)$. Hence $A$ will now be given by

$$
A=-\frac{2}{\pi} \int_{0}^{\infty} B_{0}^{2}(p) \frac{K_{0}(p c)}{I_{0}(p c)} d p
$$

where

$$
I_{0}(p \rho) e^{i p z}=\sum_{r=0}^{\infty} B_{r}(p) P_{r}(\zeta) P_{r}(i \zeta) .
$$

Equation (18) is a formal identity between two solutions of Laplace's equation both non singular at the origin, it is thus formally valid for all $p, z, \xi, \zeta$ and hence setting $\zeta=-i$ gives

$$
e^{p a e \xi}=\sum_{r=0}^{\infty} B_{r}(p) P_{r}(\xi)
$$

and hence

$$
B_{0}=\frac{1}{p a e} \sinh \text { pae. }
$$

Equations (17) and (19) may be combined to give an exact expression for $A$ but since equation (8) will only be valid to $O\left(\varepsilon_{1}^{6}\right)$ only terms up to $O\left(\varepsilon_{1}^{4} / c\right)$ need be retained in the expression for $A$. Thus the appropriate value to be used in equation (8) is

$$
A=\frac{-2}{\pi c}\left\{I(0)+\frac{\left(e \varepsilon_{1}\right)^{2} I(2)}{9}+\frac{2}{225}\left(e \varepsilon_{1}\right)^{4} I(4)\right\}
$$

numerical values of the I's have been obtained by Smythe (2). It now follows from equations (8) and (20) and the known result for the capacity of an oblate spheroid that the capacity $C$ of the conductor formed by the spheroid and the 
cylinder is given by

$$
C=\frac{a e}{\sin ^{-1} e}\left[1-\frac{2 \varepsilon_{1} e}{\pi \sin ^{-1} e}\left\{I(0)+\frac{1}{9}\left(e \varepsilon_{1}\right)^{2} \frac{I(2)}{9}+\frac{2}{225}\left(e \varepsilon_{1}\right)^{4} I(4)\right\}\right]^{-1}+O\left(\varepsilon_{1}^{6}\right) .
$$

For the particular case of a disc the result of equation (21) agrees with one previously obtained by the author when it is taken into account that due to an arithmetical error $17 / 360$ in the expression for $C$ in (4) should be $16 / 360$ (i.e. $2 / 45$ ). For the case of a spheroid with $e=\sqrt{ } 3 / 2$ detailed numerical calculations for $C$ have been carried out by Smythe (2) and the difference between Smythe's result and that of equation (21) is less than 2 in $10^{6}$ for $\varepsilon_{1}=0.5$ and the percentage error is less than $0 \cdot 7$ for $\varepsilon_{1}=0 \cdot 7$.

The analysis for a prolate spheroid of semi major axis $a$ and eccentricity $e$ almost exactly duplicates that for the oblate spheroid. $A$ is again given by equation (17) where now

and hence

$$
B_{0}(p)=\frac{1}{p a e} \sin p a e
$$

$$
C=\frac{2 a e}{\log \left(\frac{1+e}{1-e}\right)}\left[1-\frac{4 a e}{\pi \log \left(\frac{1+e}{1-e}\right)}\left\{I(0)-\frac{1}{9}\left(e \varepsilon_{1}\right)^{2} I(2)+\frac{2}{225}\left(e \varepsilon_{1}\right)^{4} I(4)\right\}\right]+O\left(\varepsilon_{1}\right) \text {. }
$$

The numerical values of $C$ for $e=\sqrt{ } 3 / 2$ were compared with those obtained by Smythe, the difference between the values was less than 2 in $10^{4}$ for $\varepsilon_{1}=0.4$ and the percentage error was found to be less than 0.5 for $\varepsilon_{1}=0.8$.

The corresponding result for an oblate spheroid placed with its centre midway between two parallel planes and its axis of symmetry normal to the planes may be written down almost immediately from equations (10), (11) and (17). The appropriate value for $A$ in equation (8) is now given by

$$
A=-\int_{0}^{\infty} \frac{e^{-p d}}{\cosh p d} \frac{\sin ^{2} p a e}{(p a e)^{2}} d p,
$$

and expansion in powers of $\varepsilon_{2}(=a / d)$ gives

$$
A=\frac{-1}{d}\left[\log 2-\frac{1}{8}\left(e \varepsilon_{2}\right)^{2} \zeta(3)+\frac{1}{16}\left(e \varepsilon_{2}\right)^{4} \zeta(5)\right]+O\left(\varepsilon_{2}^{5} / d\right) .
$$

For the particular case of a circular disc of radius $a$ equations (8) and (27) give

$$
\begin{aligned}
& \frac{\pi C}{2 a}=1+\frac{2}{\pi} \log 2 \varepsilon_{2}+\left(\frac{2 \varepsilon_{2}}{\pi} \log 2\right)^{2}+\varepsilon_{2}^{3}\left\{8\left(\frac{\log 2}{\pi}\right)-\frac{\zeta(3)}{4 \pi}\right\} \\
&+ \varepsilon_{2}^{4}\left\{\frac{-1}{\pi^{2}} \zeta(3) \log 2+\left(\frac{2 \log 2}{\pi}\right)^{4}\right\} \\
&+\varepsilon_{2}^{5}\left\{\left(\frac{2 \log 2}{\pi}\right)^{5}\right. \\
&\left.-\frac{3 \log 2 \zeta(3)}{\pi^{3}}+\frac{\zeta(5)}{8 \pi}\right\}+O\left(\varepsilon_{2}^{6}\right) .
\end{aligned}
$$


The above expression for $C$ correct up to the term in $\varepsilon_{2}^{4}$ has previously been obtained by Collins (3).

The appropriate value for $A$ in equation (8) for a prolate spheroid between two planes is obtained by replacing $e$ by $i e$ in equation (23).

\section{Application to elastostatics}

The approach of Section 2 can also be generalised to derive equations analogous to equations (6) and (7) for problems involving cracks and cavities under torsion. We consider first the general problem of an axi-symmetric crack, whose surface will be denoted by $S_{1}$, inside a circular cylinder of radius $c$. The axes of symmetry of the crack and cylinder are assumed to coincide and it is also assumed that the cylinder is maintained under torsion by a couple applied about the axis of symmetry. The definition of a crack is that adopted by Sadowsky and Sternberg (8) i.e. that $S_{1}$ separates the material and hence that there is no stress across $S_{1}$.

Polar coordinates $\rho, z, \phi$ are chosen with the $z$-axis coincident with the axis of symmetry and we then have that in Love's notation (9) the only nonvanishing stresses $\overparen{z \phi}, \overparen{\rho \phi}$ are derived from the axi-symmetric torsion function $\chi_{1}$ by

where

$$
\overparen{z \phi}=\frac{\mu}{\rho^{2}} \frac{\partial \chi_{1}}{\partial \rho}, \quad \overparen{\rho \phi}=\frac{-\mu}{\rho^{2}} \frac{\partial \chi_{1}}{\partial \rho},
$$

$$
\frac{\partial^{2} \chi_{1}}{\partial \rho^{2}}-\frac{3}{\rho} \frac{\partial \chi_{1}}{\partial \rho}+\frac{\partial^{2} \chi_{1}}{\partial z^{2}}=0
$$

and $\mu$ is the shear modulus. If the applied couple is given by $\frac{1}{2} \pi \mu \tau c^{4}$, where $\tau$ is a constant, then it follows from the formulations of Ling (6) and Collins (5) that $\chi_{1}=\chi+\frac{1}{2} \tau \rho^{4}$, where $\chi$ vanishes on the cylinder and is equal to $-\frac{1}{4} \tau \rho^{4}$ on $S_{1}$. The constant $\tau$ is the angle of twist per unit length for a cylinder without a crack.

If $\chi$ is written as $\rho^{2} \psi$ then

$$
\frac{\partial^{2} \psi}{\partial \rho^{2}}+\frac{1}{\rho} \frac{\partial \psi}{\partial \rho}+\frac{\partial^{2} \psi}{\partial z^{2}}-\frac{4}{\rho^{2}} \psi=0
$$

Thus $\psi \cos 2 \phi$ is a solution of Laplace's equation vanishing on $S_{2}$ and equal to $-\frac{1}{4} \tau \rho^{2}$ on $S_{1}$. It now follows immediately from potential theory that

$$
\psi=\int_{C}\left[G_{0}^{(2)}\left(\rho_{1}, \rho_{2}, z_{1}, z_{2}\right)+2 \pi G_{1}^{(2)}\left(\rho_{1}, \rho_{2}, z_{1}, z_{2}\right)\right] \sigma\left(\rho_{1}, z_{1}\right) \rho_{1} d s, \ldots
$$

where $C$ is the bounding curve of $S_{1}$ in the meridian plane, the notation in equation (27) is that of Sections 2, 3, i.e. the suffix 1 refers to a point $P$ on $S_{1}$ and 2 to an arbitrary point $Q$. In this notation $\pi G_{0}^{(2)}$ is the coefficient of $\cos 2\left(\phi_{1}-\phi_{2}\right)$ in the Fourier expansion of the free space Green's function $G_{0}(P, Q), G_{1}^{(2)}$ is defined by equation (10) with $r=2$ and $4 \pi \sigma$ is the discontinuity in the normal derivative of $\psi$ across $C$. 
The boundary value problem for $\psi$ thus reduces to solving $-\frac{1}{4} \tau \rho_{2}^{2}=\int_{C}\left[G_{0}^{(2)}\left(\rho_{1}, \rho_{2}, z_{1}, z_{2}\right)+2 \pi G_{1}^{(2)}\left(\rho_{1}, \rho_{2}, z_{1}, z_{2}\right)\right] \sigma\left(\rho_{1}, z_{1}\right) \rho_{1} d s$, $\left(\rho_{2}, z_{2}\right)$ on $C$.

If $a$ is used to denote the maximum distance between two points of $C$ it follows from equation (28) that on $C$

$$
2 \pi G_{1}^{(2)}=\rho_{1}^{2} \rho_{2}^{2}\left\{A+G_{3}\left(\rho_{1}, \rho_{2}, z_{1}, z_{2}\right)\right\}
$$

where $G_{3}$ is $O\left(A a^{2} / c^{2}\right)$ and

where

$$
A=\frac{-3 \pi}{64 c^{5}} \beta_{4}
$$

$$
\beta_{2 r}=\frac{2^{2 r+1}}{\pi(2 r) !} \int_{0}^{\infty} v^{2 r} \frac{K_{2}(v)}{I_{2}(v)} d v
$$

and the $\beta$ 's have been tabulated by Ling up to $\beta_{24}(5)$.

By an analysis similar to that of Section 2 is now follows that equation (28) may be re-written

where

$$
-\frac{1}{4} \tau \rho_{2}^{2}=\int_{C} G_{0}^{(2)} \sigma^{\prime} \rho_{1} d s+\rho_{2}^{2} \int_{C} G_{3} \sigma^{\prime} \rho_{1} d s,
$$

and hence

$$
\sigma^{\prime}=\sigma\left[1+\frac{4 A}{\tau} \int_{C} \rho_{1}^{3} \sigma d s\right]^{-1}
$$

$$
\int_{C} \rho_{1}^{3} \sigma d s=\int_{C} \rho_{1}^{3} \sigma^{\prime} d s \cdot\left[1-\frac{4 A}{\tau} \int_{C} \rho_{1}^{3} \sigma^{\prime} d s\right]^{-1} .
$$

[For notational convenience the dependence of $\sigma^{\prime}, G_{0}^{(2)}, G_{3}$ on $\rho_{1}, z_{1}$, etc., is not demonstrated explicitly in the above equations.]

Equations (29) and (30) and simple dimensional considerations show that the ratio of the second integral on the right hand side of equation (31) to the first is $O\left(\varepsilon^{7}\right)$ where $\varepsilon=a / c$. Hence $\sigma^{\prime}=\sigma_{0}\left[1+O\left(\varepsilon^{7}\right)\right]$ where

$$
-\frac{1}{4} \tau \rho_{2}^{2}=\int_{C} G_{0}^{(2)} \rho_{1} \sigma_{0} d s
$$

It also follows from equation (33) that

$$
\int_{C} \rho_{1}^{3} \sigma d s=\int_{C} \rho_{1}^{3} \sigma_{0} d s \cdot\left[1-\frac{4 A}{\tau} \int_{C} \rho_{1}^{3} \sigma_{0} d s\right]^{-1}+O\left(\varepsilon^{7}\right) .
$$

Equation (34) is the integral equation for a crack in an infinite medium and it will be assumed that the solution of this problem can be found in a convenient form. Equations (32) and (35) then show that the solution for a crack in a cylinder may be found up to $O\left(\varepsilon^{7}\right)$ immediately from the solution in the infinite medium. Equation (35) may be used to obtain a simple relationship between 
$E_{1}$-the loss of potential energy due to a crack in an infinite medium and $E_{2}$ the corresponding quantity due to a crack in the cylinder. From equation (35) and the expression for the energy derived in (10) it follows that

$$
E_{2}=E_{1}\left[1+\frac{A E_{1}}{\mu \tau^{2} \pi^{2}}\right]^{-1}+O\left(\varepsilon^{7}\right)
$$

For the cases of spherical and penny shaped cracks equation (36) agrees with a result derived by Shail (11)

Another quantity of physical interest which can be expressed in terms of the left hand side of equation (35) is $\omega_{1}$, the increase in the angle of twist due to the presence of the crack in the cylinder. We have that (6)

$$
\omega_{1}=\frac{1}{\rho_{2}^{3}} \frac{\partial}{\partial \rho_{2}}\left[\rho_{2}^{2} \int_{-\infty}^{\infty} \psi d z_{2}\right]
$$

where $\psi$ is given by equation (27). After reduction this gives

$$
\omega_{1}=\frac{-4 \pi}{c^{4}} \int_{C} \rho_{1}^{3} \sigma d s .
$$

If $G_{1}^{(2)}$ is defined by equation (11) with $r=2$ then equation (28) is the appropriate integral equation for a crack $S_{1}$ in a plate of thickness $2 d$ with the axis of symmetry of the crack normal to the faces of the plate. In this case the plate is maintained under torsion by a shear stress $\mu \tau \rho_{2}$ applied over its faces. Equations (32) to (36) will once more be valid if $A$ is now defined by

$$
A=\frac{-45 \pi}{1024 d^{5}} \zeta(5),
$$

where $\zeta(s)$ is the Riemann Zeta function. For spherical and penny shaped cracks the ratio $E_{2} / E_{1}$ derived from equation (36) and (38) agrees with a corresponding result derived by Shail (11). As for the electrostatic problems it is possible to improve the accuracy of equation (35) for the particulars cases of spheroidal cavities in a cylinder or a thick plate. These particular cases will now be examined in the following section.

\section{Problems involving spheroidal cavities}

We consider first an oblate spheroidal cavity in a circular cylinder of radius $c$. The centre of the spheroid is assumed to be on the axis of the cylinder and its axis of symmetry assumed to coincide with the cylinder axis. Equations (27) and (28) will still be valid provided that $4 \pi \sigma$ is now interpreted as

$$
\frac{\partial}{\partial n}\left[\psi+\frac{1}{4} \tau \rho^{2}\right]
$$

where $\partial / \partial n$ denotes the outward normal derivative on $C$.

By a simple extension of the analysis of Section 3 it can be shown that, provided $A$ is the coefficient of $\rho_{1}^{2} \rho_{2}^{2}$ in the expansion of $2 \pi G_{1}^{(2)}$ on the spheroid 
as a series in $P_{n}^{(2)}\left(\check{\zeta}_{1}\right) P_{n}^{(2)}\left(\xi_{2}\right), \sigma^{\prime}=\sigma_{0}\left[1+O\left(\varepsilon^{14}\right)\right]$ and hence that equation (35) is valid with an error term of $O\left(\varepsilon^{14}\right)$.

Thus $A$ will now be given by

where

$$
A=-324 \int_{0}^{\infty} \frac{B_{2}^{2}(p) K_{2}(p c)}{I_{2}(p c)} d p
$$

$$
e^{i p z} I_{2}(p \rho)=\sum_{r=2}^{\infty}(a e)^{2} B_{r}(p) P_{r}^{(2)}(\check{\zeta}) P_{r}^{(2)}(i \zeta)
$$

It follows from equation (40) (letting $\xi \rightarrow-i$ ) that

$$
B_{2}=\frac{5 p^{2}}{384} \int_{-1}^{1}\left(1-v^{2}\right)^{2} e^{-a e p v} d v .
$$

The integral in the above expression is elementary but the subsequent manipulation is considerably simplified by re-writing the equation as

$$
B_{2}=\frac{5 p^{2}}{384} \times \frac{2^{\frac{3}{2}} \sqrt{ } \pi}{(p a e)^{\frac{3}{2}}} I_{\frac{5}{2}}(p a e)
$$

Thus from equations (39) and (41)

$$
A=\frac{-225 \pi}{32 c^{5}} \int_{0}^{\infty} \frac{K_{2}(v)}{I_{2}(v)} v^{4} \frac{I_{\frac{3}{2}}^{2}(\varepsilon v) d v}{(\varepsilon v)^{5}}
$$

Equation (42) is an exact expression for $A$ but, as equation (35) is only valid up to $O\left(\varepsilon^{14}\right)$, the appropriate form of $A$ for use in equation (35) will be a power series in $\varepsilon$. The series expansion for $I_{n}^{2}(12)$ considerably simplifies the manipulations involved in the derivation of the series for $A$. The final result is

$$
\begin{aligned}
A=\frac{-9 \pi}{c^{5}}\left\{\frac{\beta_{4}}{192}+\frac{5 \beta_{6}(\varepsilon e)^{2}}{896}+\frac{5 \beta_{8}(\varepsilon e)^{4}}{1008}+\right. & \frac{125 \beta_{10}(e \varepsilon)^{6}}{29568} \\
& \left.+\frac{750}{91} \beta_{12}(e \varepsilon)^{8}+O\left(\varepsilon^{10}\right)\right\}
\end{aligned}
$$

The appropriate value of $A$ for a prolate spheroid is obtained from equation (42) by replacing $e$ by $i e$. It follows from an examination of the analysis leading to equation (43) and a comparison of equations (10) and (11) that the appropriate value of $A$ for an oblate or prolate spheroid in a thick plate may be deduced from the corresponding result for a spheroid in a cylinder by replacing $\varepsilon$ by $i a / d$ and $\beta_{2 r}$ by $\left(1-2^{-2 r}\right) \zeta(2 r+1)$.

For the case of a penny-shaped crack in a circular cylinder the ratio $E_{2} / E_{1}$ has been calculated by Collins (5), who reduces the problem to a Fredholm integral equation of the second kind. Collins obtains the ratio correct to $O\left(\varepsilon^{12}\right)$ and, to this order, his result agrees with that obtained from equations (36) and (43). Corresponding agreement is also obtained for a penny-shaped crack in a thick plate.

Numerical values of $\omega_{1}$ obtained from equations (35) and (37) with $A$ 
defined by equation (43) [with $e=0$ ] show complete agreement with the numerical values of $\omega_{1}$ obtained by Ling (6) for a spherical cavity in a cylinder for $\varepsilon \leqq 0 \cdot 5$. The discrepancy between the results of equations (35) and (37) and those of Ling is less than 3 in $10^{5}$ for $\varepsilon=2 / 3$.

The procedure can also be applied to the problem of a spheroidal cavity in a thick plate of thickness $2 d$ whose faces are kept fixed when the cavity is rotated about its axis of symmetry through a small angle $\alpha$. It is assumed that the axis of symmetry is normal to the faces of the plate and that the centre of the spheroid is midway between the faces of the plate. It can be shown that, for an oblate spheroid, the applied couple $G$ necessary to produce the displacement is given by

$$
G=\frac{G_{0}}{\left[1+\frac{A G_{0}}{8 \pi \mu \alpha}\right]}+O\left(\varepsilon^{10}\right),
$$

where $\varepsilon=a / d, G_{0}$ is the corresponding couple for an infinite medium and

$$
A=\frac{-\pi}{2 d^{3}}\left[\frac{3 \zeta(3)}{8}-\frac{9(\varepsilon e)^{2} \zeta(5)}{32}+\frac{729(e \varepsilon)^{7} \zeta(7)}{3200}+\frac{51(e \varepsilon)^{6} \zeta(9)}{320}\right] .
$$

For the case of a sphere and $\varepsilon=\frac{1}{2}$ expressions (44) and (45) give a value for $G$ which agrees exactly with that given by Fox (7).

\section{Possible extensions}

The general procedure of the preceding sections can be easily extended to solve the general non-axisymmetric problems for spheroids in cylinders and between parallel planes. Two particular cases have been examined in detail and the problem treated briefly at the end of Section 5 can be reduced to the problem of finding a function $\psi$ satisfying prescribed boundary conditions and such that $\psi \cos \phi$ is a solution of Laplace's equations. Problems where the normal derivative of a potential function are prescribed on $S_{2}$ may also be treated similarly.

The present work has been concerned with the simple determination of some significant physical parameter in the various problems treated but it is of interest to note that the integral equation formulation of Section $\mathbf{2}$ may be adapted to obtain more detailed properties of the solution without recourse to elaborate perturbation procedures, etc. This approach will now be examined very briefly for the particular case of the sphere placed symmetrically in a cylinder. It follows from equation (15) that equation (3) becomes

$$
\begin{aligned}
1=\int_{S_{1}} G_{0}(P, Q) \sigma(P) d S-\frac{1}{\mathcal{C}} \operatorname{Re}\left\{\sum_{n}\right. & =0 \\
& \left.\int_{S_{1}} \sum_{n=0}^{\infty} \varepsilon_{1}^{n} A_{n} P_{n}\left(\cos \theta_{n}\right)\right\}
\end{aligned}
$$


The equation

$$
P_{n}\left(\cos \theta_{2}\right)=\int_{S_{1}} \sigma G_{0}(P, Q) d S
$$

is the equation occurring in the electrostatic boundary value problem for $S_{1}$ when the prescribed potential is $P_{n}\left(\cos \theta_{2}\right)$. This problem is soluble by standard methods giving $\sigma=B_{n} P_{n}\left(\cos \theta_{2}\right)$ where $B_{n}$ may be found. Thus equation (46) may be re-written as

$B_{0}=\sigma-\int_{S_{1}} \sigma(P)\left\{\frac{1}{c} \operatorname{Re}\left[\sum_{n=0}^{\infty} \varepsilon_{1}^{n} A_{n} B_{n} P_{n}\left(\cos \theta_{2}\right)\right]\left[\sum_{n=0}^{\infty} \varepsilon_{1}^{n} A_{n} P_{n}\left(\cos \theta_{1}\right)\right]\right\} d S .(47)$

Equation (47) is a Fredholm integral equation of the second kind for $\sigma$ and may be solved by iteration. The above approach is not the most suitable for the sphere as it has been shown (4) that a simpler governing integral equation can be obtained for a function $S$ which is directly related to $\sigma$. Equations analogous to equations (46) and (47) can be derived for the problem of a spheroid in a cylinder and a formal solution could be obtained. The kernels of these equations will necessarily be complicated and it does not seem possible to use the technique of (4) to reduce them to a simpler form.

\section{REFERENCES}

(1) J. C. CoOKe, Z.A.M.M., 42 (1962), 305.

(2) W. R. Sмythe, J. Math. Phys., 4 (1963), 833.

(3) W. D. Collins, Proc. Edin. Math. Soc., 12 (1960), 95.

(4) W. E. Williams, Z.A.M.P., 13 (1962), 133.

(5) W. D. Collins, Mathematika, 9 (1962), 25.

(6) C. B. LiNG, Quart. App. Math., 10 (1952), 149.

(7) N. Fox, Proc. Lond. Math. Soc., 11 (1961), 276.

(8) M. A. Sadowsky and E. Sternberg, J. App. Mech., 71 (1949), 149.

(9) A. E. H. Love, Theory of Elasticity (Cambridge, 1952).

(10) G. Weiss and L. E. Payne, J. App. Phys., 25 (1954), 1321.

(11) R. Shall, to be published in J. Math. and Mech.

(12) G. N. Watson, Theory of Bessel Functions (Cambridge, 1958).

Department of ApPlied Mathematics

THE UNIVERSITY

LIVERPOOL 